\section{P-125 RE-AUDIT ON 'DISCUSSION OF CORNEAL DONATION' WITH PEOPLE ADMITTED TO HOSPICE}

Ramesh Thulavavenkateswaran, Mike Machila, Heidi Downs. St Helena, Colchester, UK

10.1136/bmjspcare-2019-HUKNC.148

Background There is a national shortage of approximately 500 corneas per year that could be used for sight restoring transplants (NHS Blood and Transplant). 51\% of the UK adult population state that they would be willing to donate some/all of their organs when they die (NHS Blood and Transplant, 2013). Evidence shows it can bring great comfort to bereaved families.

We recognised the importance that people are given the opportunity to donate their corneas. In April 2018, as part of the hospice's admissions process, an initial screening questionnaire assessing both eligibility and clinical appropriateness (those not actively dying or deemed to lack capacity) was developed. An initial audit was completed in June 2018 which demonstrated only $8 \%(5 / 66)$ of people admitted to hospice had a discussion about corneal donation.

Aim To increase awareness of corneal donation and the opportunity to donate.

Method Following the initial audit, appropriate changes were made to the initial screening questionnaire. The medical team were provided with training and education to enable them to complete the necessary assessment with the aim of increasing the discussions around corneal donation upon admission. Over a nine month period we re-audited the care plans of those admitted.

Results

- People admitted to hospice=241;

- Initial screening questionnaire completed=62 (26\%);

- People eligible with no contraindications $=40$;

- People who were deemed clinically appropriate=29;

- People who agreed to be a donor $=16$;

- People who donated their corneas after death $=5$.

Conclusion There is an improvement on assessment for corneal donation from $8 \%$ to $26 \%$. We will continue to provide education and training to encourage more initial screening questionnaires to be completed to ensure that people can engage in these conversations where appropriate. We need to explore the factors where corneal donation is agreed but does not happen.

\section{P-126 IMPLEMENTATION OF A PATHWAY TO IMPROVE RATES OF CORNEAL DONATION WITHIN A HOSPICE SETTING: A QIP}

Rory Carrigan, Diane Drain, Samantha Wilks, Amanda Williams, Thomas Carter, Pia Amsler. Saint Francis Hospice, Romford, UK

\subsection{6/bmjspcare-2019-HUKNC.149}

Introduction Within the UK, there is a transplant shortage of approximately 500 corneas each year. Corneal transplant is a sight-saving procedure and corneal donation can be empowering for end-of-life patients who are otherwise unable to donate their organs. We set out to improve corneal donation rates within our organisation.

Methods Prior to our intervention there was no formal guidance on the process of corneal donation or any records on organisation donation rates. We arranged specialist local training in collaboration with NHS Blood and Transplant to train and educate hospice staff on the process of corneal donation. Following this, guidelines were implemented to facilitate conversations with patients, including a guide for both staff and patients on the process of corneal donation. Staff were also afforded the opportunity to observe the corneal retrieval process to build confidence during discussions with patients and their relatives.

Results Between November 2018 and June 2019, corneal donation was discussed with $75 \%$ of patients admitted to the inpatient unit, resulting in 30 successful corneal donations. In addition, all staff members consulted, including doctors, nursing staff and clinical support workers felt more empowered to discuss corneal donation with patients. Consistent documentation using a traffic-light system, where patients for corneal donation (green), considering corneal donation (yellow), or not for corneal donation (red) helped promote discussion and awareness. Daily patient reports emailed to key project members helped ensure all staff were kept updated with patient wishes.

Conclusions These preliminary results suggest that our intervention has been successful, resulting in 30 donations, which has the potential to restore the sight of over 300 people. Data will continue to be collected and future aims include expanding the project into hospice outpatient settings. To facilitate this, corneal donation champions have been nominated to raise the awareness of corneal donation across the organisation.

\section{P-127 'ORCHESTRA OUTREACH'- A PARTNERSHIP BETWEEN A HOSPICE AND A WORLD-RENOWNED CONCERT ORCHESTRA}

Emily Macieira Santos. Peace Hospice Care, Watford, UK

10.1136/bmjspcare-2019-HUKNC.150

Background The hospice's services are orientated towards a self-management agenda. As part of this we support service users to access community experiences to increase their social interaction and feelings of empowerment. This also raises community awareness of hospice services.

Aims Music can enhance and maintain wellbeing in adults with diagnosed conditions. Our Orchestra Outreach initiative is an example of innovative partnership working, empowering people to access creative opportunities, leading to increased wellbeing. The hospice is situated near to an auditorium where a world-renowned orchestra rehearses. The orchestra manager invites our patients, carers, volunteers and staff to attend their rehearsals. This opens up wellbeing opportunities for all.

Method Hospice staff liaise with the orchestra manager to get the rehearsal programme which is then circulated through various mediums. Staff accompany the group initially and then members of the group come and go as they please, thus encouraging self-management decisions. Hospice staff record who has attended each performance.

Results From October 2018 - May 2019, 70 members of our community have attended performances. Participants reported feelings of enjoyment and relaxation, as well commenting on the special delight that comes with gaining a unique insight into that which is usually hidden from view. Staff members have talked enthusiastically about being immersed in music 